\section{Opening up Rosetta}

\section{By Lev Osherovich, Senior Writer}

Merck \& Co. Inc.'s soon-to-be shuttered Rosetta Inpharmatics Inc. unit is to be reborn as Sage, a not-for-profit institute that aims to provide precompetitive open access to the pharma's integrative genomics data set. This development takes on added significance now that newly published experiments help to validate Rosetta's intensive computational approach to metabolic disease target identification.

The mouse study in Nature Genetics shows that eight proteins involved in various aspects of metabolism and immunity could potentially be targeted to treat obesity and dyslipidemia. ${ }^{1}$ The paper makes the most compelling case yet for the Rosetta approach, which involves predicting key genetic players in disease by sifting through massive sets of gene expression, protein-protein interactions, SNPs and quantifiable physiological traits data.

Merck is now winding Rosetta down, and the team behind the study plans to spin the division out as Sage. The goal is to bring academic biologists and computer scientists, plus industry pharmacogenomics researchers, together in a "precompetitive space," said Eric Schadt, the study's coauthor and formerly executive scientific director of Rosetta and now VP and CSO of Sage.

The key idea behind Sage, said Schadt, is to make Merck's previously proprietary data accessible to all interested parties without any IP strings attached. Open access to genomic data could move the discovery side of the industry away from a competitive model and toward consortium-style collaborations with academia, Schadt told SciBX.

Schadt and Rosetta founder Stephen Friend plan to launch Sage in July as a not-for-profit organization to continue Rosetta's collaborations with academia and industry and to build open-access software for genomic data analysis. Friend will be president and CEO of Sage.

Merck is backing the project by donating computers, and it plans to release most of the genomic data gathered by Rosetta into the public domain via Sage.

Although it will be another 3-5 years before Internet users will have full access to Sage, the team is already working with academics in three U.S. universities to open up Merck's database to new collaborators.

\section{Fat city}

Although previous studies by the Rosetta team had pointed to genes likely to play roles in obesity and diabetes in mice and humans, ${ }^{2,3}$ broad experimental proof had been lacking until now.

"This paper pulls together the validation that the algorithm is really quite predictive of biological significance on a gene-by-gene basis," said
Thomas Drake, professor of pathology, laboratory medicine and clinical chemistry at the University of California, Los Angeles, and the senior author of the Nature Genetics study. "This opens up the possibility of identifying many more significant genes than you could with classical genetic approaches."

Classical approaches involve identifying mutants based on their phenotypes rather than trying to predict phenotype from computational models.

In previous studies, Rosetta and UCLA teams jointly analyzed integrative genomic data to come up with a list of the genes most likely to influence obesity and metabolic disease. ${ }^{4}$ In the new study, the researchers made knockout or transgenic mice with altered expression of the nine statistically strongest candidate genes.

The team then fed high-fat diets to the mutant mice and measured their body weight, plasma lipid composition and fat-to-muscle ratio. Eight of the nine mutant strains displayed abnormal fat-related phenotypes.

For example, mice with extra copies of zinc finger protein 90 homolog (ZFP90) and $\beta$-lactamase ( $L A C T B$ ), as well as mice missing complement component 3 a receptor 1 (C3AR1; C3AR) and lipoprotein lipase (LPL), had higher overall fat levels than wild-type controls.

ZFP90 and C3AR were not previously known to affect lipid metabolism, and the role of LACTB in normal lipid metabolism was poorly understood.

Conversely, the team found that mice overexpressing growth arrest-specific 7 (GAS7) or glutathione peroxidase 3 plasma (GPX3) and mice lacking NADP-dependent malic enzyme (ME1) or transforming growth factor- $\beta$ receptor II (TGFBR2) had lower fat-related profiles than wild-type controls.

Altogether, the findings suggest ZFP90, ME1, LACTB and TGFBR2 could be antagonized to treat obesity, whereas $C 3 A R, L P L, G A S 7$ and $G P X 3$ could be agonized to achieve a similar effect.

UCLA's Drake believes the next step will be to choose targets from this list for pharmacological discovery and development. Indeed, most of the proteins on the list are either enzymes or receptors and are considered druggable.

Merck did not disclose whether it was pursuing any of the targets in the study.

Drake also thinks the approach won't be limited to obesity and expects that the technique could be used to identify metabolic genes involved in cancer. "There are close relationships between all of these basic body response mechanisms and cancer metabolism," he said.

Schadt added that the method's eight of nine success rate at predicting which mutations lead to obesity phenotypes could convince companies to dive headlong into higher-risk projects.

Typically, drug development doesn't begin until a large body of preclinical data provides a rationale for targeting a protein. However, Schadt believes that it may be worthwhile to launch small molecule screens against proteins pegged as key players based on computer modeling alone.

"If our predictions are achieving this kind of accuracy, you might be willing to place bigger bets without doing the animal [knockout] experiments," he said. 
Jeff Shrager, CTO of computational biology company CollabRx Inc. and associate professor of symbolic systems at Stanford University, said the Nature Genetics study highlights the statistical power of Rosetta's approach.

"They're doing a very good job of putting numbers to their results. They can tell you not just what molecules are relevant, but can put statistical values on their predictions," he said.

However, Shrager cautioned that the genes chosen for in vivo validation in the Nature Genetics study may have been skewed toward enzymes with plausible biological roles in lipid metabolism due to overfitting of the computer models. If both computation and human expertise influenced the decision about which genes to study, the method may not be as useful for more poorly understood areas of biology, he said.

Shrager noted that the study lacked negative controls of mouse knockouts or transgenics chosen at random or from among genes that the computer deemed unlikely to be relevant to obesity.

Drake maintained that it was impractical to perform an extensive metabolic analysis on negative controls aside from wild-type mice and said it was highly unlikely that the eight of nine correct predictions were due to chance.

He said no new IP had been filed on the discoveries in the Nature Genetics study. A Merck
"Doing the disease modeling work in a precompetitive space will free up resources for the real business of developing therapeutics."

-Stephen Friend, Sage academic and industry researchers to use Sage's data to make computational models of disease mechanisms and eventually add their own genomic data to the core dataset from Merck.

"This data set is only $1 \%$ of the biology for which you could potentially want to make models," said Friend, who said additional data would increase the predictive power of the database and could yield better results than solo in-house efforts.

"Currently, information generated in proprietary zones is smaller and less sufficient for models of disease than something in a larger public space like Sage," he said. "Doing the disease modeling work in a precompetitive space will free up resources for the real business of developing therapeutics."

Once Sage's software interface is in place, academic and industry researchers will be able to query and contribute to a large collection of data to generate testable hypotheses that will lead to publications, IP and, potentially, products (see Figure 1, "New value chain in genomics").

The Sage system is "going to be free and in the public domain," akin to the sequence data from the Human Genome Project, said John Wilbanks, executive director of the not-forprofit advocacy group Science Commons and a Sage $\mathrm{SAB}$ member.

Wilbanks expects most users to initially look at Merck's data without contributing their own spokesperson said some of the methods used in the study are covered by previous patents, which the company plans to make available to Sage.

\section{Genomics clearinghouse}

Schadt and Friend now plan to make the methodology used in the Nature Genetics study available to all Sage members. The goal of the project, said Friend, is to allow any researcher to probe Merck's database for interesting genetic interactions in other disease areas.

"Seven years, \$100 million and a lot of data in metabolism have shown us that we can build rather simple models to allow prediction of targets with real effects," said Friend.

Rosetta was acquired by Merck in 2001, and the pharma invested heavily in building the computers needed to perform Rosetta's integrative genomic analyses. Although Rosetta went on to identify genetic networks that underlie metabolic disease in animal models and humans, Merck announced plans to shutter Rosetta's research division as part of a 2008 restructuring. Rosetta Biosoftware, a separate Merck subsidiary that sells genomic analysis tools, will continue to operate.

Sage has already raised $\$ 5$ million via angel investors and hopes to receive more funding through $\mathrm{NIH}$ and disease foundation grants.

Schadt and Friend plan to relocate the core of Rosetta's staff to three academic centers: the Fred Hutchinson Cancer Research Center, Yale University and another yet-to-be-determined academic institution on the West coast. Once settled, Sage will spend 3-5 years developing software tools to rework Merck's data into a more user-friendly, standardized form and put it on the Internet.

In the interim, researchers keen to comb Merck's data will need to collaborate with Sage's academic staff at the three host universities, according to Schadt.

Friend and Schadt hope the Nature Genetics study will entice observations to the common pool. However, users will get the most out of the analysis when they upload their own data sets and compare them against Merck's findings, he said.

\section{The value proposition}

Keith Yamamoto, professor of cellular and molecular pharmacology and executive vice dean for research at the University of California, San Francisco, thinks Sage is "the vanguard of a broader agenda to change academic-industry relations."

According to Yamamoto, the complexity of genomic data exceeds the capacity of any one company or academic group to comprehend it, making data sharing and collaboration essential for further progress.

"Consortia of academic and industry groups can take on complicated preclinical problems and biological processes that could yield benefits to both sides," he said. "These could include truly predicting toxicology, making better animal models or-as Schadt is doing-developing predictive models."

William Chin, SVP of discovery research and clinical investigation at Eli Lilly and Co., said that pharmas will need to cooperate to understand the complex underpinnings of disease, but doing so will require changes in corporate culture to encourage data sharing.

"In fully integrated pharmaceutical companies, the focus is on control and ownership," he said. "But we now realize that we don't need to own everything."

Chin said that because Sage is still in its embryonic stages, Lilly is not yet committing any resources to the project. He did say the company would be willing to play if the Sage team can demonstrate a usable product.

"Details on contributing data will still require working out, but philosophically we would want to go down this road," said Chin. 
Figure 1. New value chain in genomics. According to the founders of Sage, an open-access genomics institute spun out of Merck \& Co. Inc., industry and academic researchers will be able to access Sage's database of 'precompetitive' gene expression data and other genomic information donated by Merck. But the ultimate richness of the data set will hinge on persuading other users to add to the database. Sage hopes the data will enable researchers to generate, test and publish papers on new hypotheses. The founders also expect new IP to be generated as scientists inside academia and corporations create new products inspired by the data.

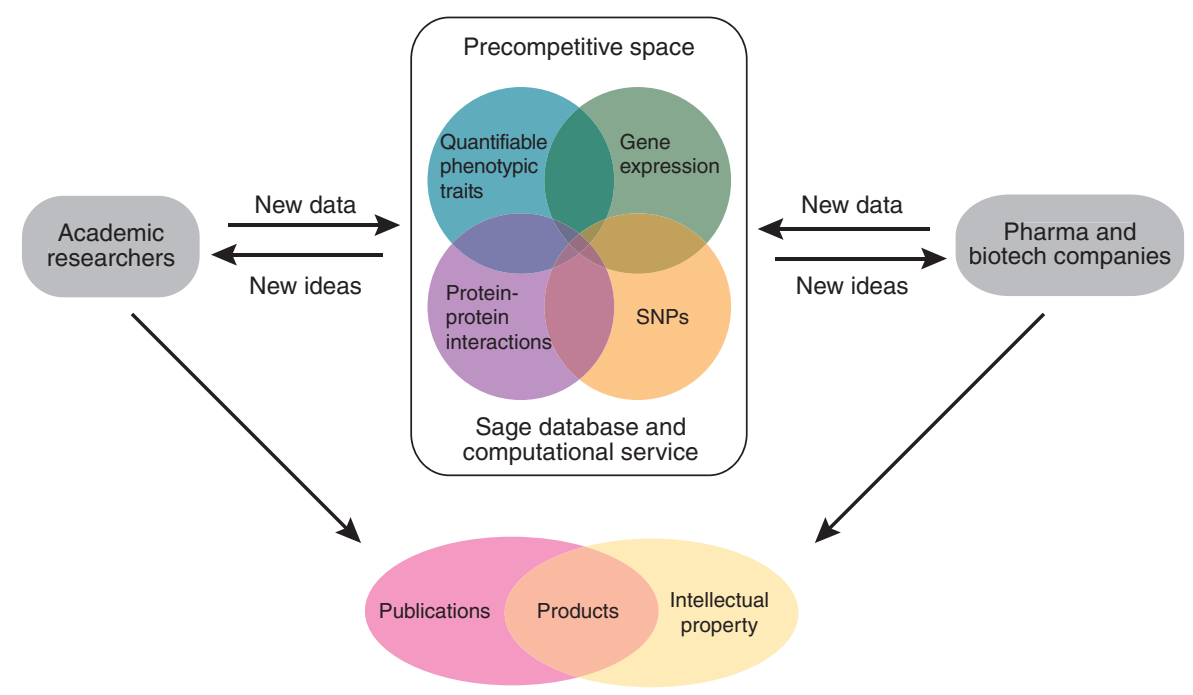

Anthony Ford-Hutchinson, SVP and franchise head at Merck, said the company isn't really giving up anything by opening its database to public users.

"When you discover targets, there really isn't much defensible IP around it," he said. "The proof will be when people start generating results from the data."

"We're hoping that Sage provides a platform to generate IP, not to have IP itself," said Friend. "Merck is transferring a significant amount of data from which it has filed IP, but another group looking at the identical information could have different interpretations."

CollabRx's Shrager was less sanguine, however, suggesting Merck may have already picked over the data for relevant IP opportunities. "If it's so useful, why is Merck putting it out the door?" he said.

Friend disagreed. "You can bet that the majority of discoveries of value are still there" in the data set, he said.

CollabRx does plan to include access to Sage as part of its webbased collaborative biocomputing software, which is in beta testing.

Osherovich, L. SciBX 2(14); doi:10.1038/scibx.2009.561

Published online April 9, 2009

\section{REFERENCES}

1. Yang, X. et al. Nat. Genet; published online March 8, 2009; doi:10.1038/ng.325

Contact: Thomas A. Drake, University of California, Los Angeles, Calif. e-mail: tdrake@mednet.ucla.edu

2. Ghazalpour, A. et al. PLoS Genet. 2, e130; published online July 5, 2006; doi:10.1371/journal.pgen.0020130

3. Chen, Y. et al. Nature 452, 429-435 (2008)

4. Schadt, E.E. et al. Nat. Genet. 37, 710-717 (2005)

\section{COMPANIES AND INSTITUTIONS MENTIONED}

CollabRx Inc., Palo Alto, Calif.

Eli Lilly and Co. (NYSE:LLY), Indianapolis, Ind.

Fred Hutchinson Cancer Research Center, Seattle, Wash. Merck \& Co. Inc. (NYSE:MRK), Whitehouse Station, N.J.

National Institutes of Health, Bethesda, Md.

Rosetta Inpharmatics Inc. (NASDAQ:RSTA), Kirkland, Wash.

Sage, Seattle, Wash.

Science Commons, San Francisco, Calif.

Stanford University, Stanford, Calif.

University of California, Los Angeles, Calif.

University of California, San Francisco, Calif.

Yale University, New Haven, Conn. 